Psychological Medicine

cambridge.org/psm

\section{Erratum}

Cite this article: Aggensteiner Pascal-M et al (2023). The effects of callous-unemotional traits and aggression subtypes on amygdala activity in response to negative faces ERRATUM. Psychological Medicine 53, 304-304. https://doi.org/10.1017/S0033291720003049

First published online: 28 August 2020

\title{
The effects of callous-unemotional traits and aggression subtypes on amygdala activity in response to negative faces - ERRATUM
}

Pascal-M Aggensteiner (D), Nathalie E. Holz, Boris W. Böttinger, Sarah Baumeister, Sarah Hohmann, Julia E. Werhahn, Jilly Naaijen, Shahrzad Ilbegi, Jeffrey C. Glennon, Pieter J. Hoekstra, Andrea Dietrich, Renee Kleine Deters, Melanie C. Saam, Ulrike M. E. Schulze, David J. Lythgoe, Arjun Sethi, Michael C. Craig, Mathilde Mastroianni, Ilyas Sagar-Ouriaghli, Paramala J. Santosh, Mireia Rosa, Nuria Bargallo, Josefina Castro-Fornieles, Celso Arango, Maria J. Penzol, Jorge Vidal, Barbara Franke, Marcel P. Zwiers, Jan K. Buitelaar, Susanne Walitza, Tobias Banaschewski and Daniel Brandeis

doi: 10.1017/S0033291720002111, Published online by Cambridge University Press, 06 July 2020

The above mentioned article was published in Psychological Medicine with an error in the conclusion. The correct conclusion is written below:

\section{Conclusion}

In summary, this large study compared children and adolescents with aggression-related problems v. TD during an fMRI emotional face-matching task, investigating the role of subtypes of aggression and CU traits. Overall, children and adolescents with high aggression showed amygdala hyperactivity in emotion and face processing areas, particularly in the subgroup with low CU traits. In contrast, in those with high CU traits, amygdala hypoactivity was observed. Our findings underline the importance to specify subtypes and CU traits in aggression-related disorders, using brain-based evidence and therefore providing a possible biomarker, which could be used for improved diagnostics and personalized treatment.

Cambridge University Press apologises for this error.

\section{Reference}

Aggensteiner, P., Holz, N., Böttinger, B., Baumeister, S., Hohmann, S., Werhahn, J., . . Brandeis, D. (2020). The effects of callous-unemotional traits and aggression subtypes on amygdala activity in response to negative faces. Psychological Medicine, 1-9. doi:10.1017/S0033291720002111 